Revisión de tema

\title{
Anticoagulación oral en el adulto mayor frágil con fibrilación auricular
}

\section{Oral Anticoagulants in the Frail Elderly with Atrial Fibrillation \\ Anticoagulação oral nas pessoas idosas com fibrilhação auricular}

\author{
Miguel Oswaldo Cadena-Sanabria, MD., Esp.* \\ Oscar Mariano Pinto-Saavedra, MD., Esp. ** \\ Juan Fernando Contreras-Valero, MD. *** \\ Gustavo Eduardo Ortega-Ramírez, MD. *** \\ Heider Alexis Bautista-Mier, MD. ****
}

\begin{abstract}
Resumen
Introducción: El envejecimiento incrementa el riesgo de trombosis y fenómenos embólicos, trae cambios fisiológicos y comorbilidades como la fibrilación auricular que hacen complejo el inicio y la seguridad de la anticoagulación. A pesar de la mayor disponibilidad de clínicas de anticoagulación y nuevos anticoagulantes orales, es bajo el porcentaje de adultos mayores que están anticoagulados aunque tengan indicación plena y ausencia de contraindicaciones. Objetivo: Revisar las principales estrategias para predecir y disminuir el riesgo de sangrado con el empleo de anticoagulantes orales en pacientes adultos mayores frágiles. Metodología: Se realizó una búsqueda sistemática sobre estudios que evaluaran la seguridad y eficacia de anticoagulantes orales en pacientes con fibrilación auricular en adultos mayores de 65 años, y una búsqueda de estudios sobre el síndrome de fragilidad y su impacto en el adulto mayor anticoagulado. Resultados y Discusión: Se ha denominado fragilidad al porcentaje de adultos mayores que presentan un mayor
\end{abstract}

deterioro de sus sistemas biológico, físico y cognitivo, conduciendo a una mayor probabilidad de desenlaces adversos en salud, discapacidad y muerte. Se han estudiado características clínicas que permiten identificar pacientes como frágiles; el fenotipo de fragilidad de Linda Fried evalúa esas características; además, existen estrategias de monitoreo de los anticoagulantes orales que deben ser conocidos para minimizar el riesgo de eventos adversos. Conclusiones: el anciano frágil presenta factores de riesgo que probablemente afectan la eficacia y seguridad de la terapia con anticoagulantes orales. Las guías clínicas existentes no proveen la suficiente evidencia y no consideran de manera multidimensional al paciente geriátrico. [Cadena-Sanabria MO, Pinto-Saavedra OM, Contreras-Valero JF, Ortega-Ramírez GE, Bautista-Mier HA. Anticoagulación oral en el adulto mayor frágil con fibrilación auricular. MedUNAB 2017; 19(3): 221-229]

Palabras clave: Fibrilación Atrial; Anciano Frágil; Anticoagulantes; Efectos Colaterales y Reacciones Adversas Relacionados con Medicamentos; Geriatría.

\footnotetext{
* Médico especialista en Medicina Interna y Geriatría. Unidad de Geriatría, Servicio de Medicina Interna, Fundación Oftalmológica de Santander Carlos Ardila Lülle (FOSCAL), Floridablanca, Colombia. Docente asociado programa de Medicina de la Universidad Autónoma de Bucaramanga (UNAB), Bucaramanga, Colombia. Docente auxiliar, Universidad Industrial de Santander (UIS), Bucaramanga, Colombia.

** Médico especialista en Medicina Interna. Universidad Autónoma de Bucaramanga(UNAB), Bucaramanga, Colombia

*** Médico Hospitalario. Fundación Oftalmológica de Santander Carlos Ardila Lülle (FOSCAL), Floridablanca, Colombia.

**** Médico residente de Geriatría. Universidad Nacional de Colombia. Bogotá, Colombia.
}

Correspondencia: Miguel Oswaldo Cadena-Sanabria, Transversal 154 No. 150 - 221 oficina 3-4, Floridablanca, Santander, Colombia. Correo electrónico: mcadena341@unab.edu.co. 


\section{Abstract}

Introduction: Aging increases the risk of thrombosis and embolic phenomena. It also brings physiological changes, and comorbidities such as atrial fibrillation that makes complex the onset and safety of anti-clotting. Despite the greater availability of anti-clotting clinics and new oral anticoagulants, the percentage of elderly who are anticoagulated is low, even though they have indications and no contraindications. Objective: To review the main strategies to predict and reduce the risk of bleeding with the use of oral anti-clotting in fragile elderly patients. Methodology: A systematic search was conducted on studies evaluating the safety and efficacy of oral anticoagulants in patients with atrial fibrillation in adults older than 65 years, and a search for studies on the frailty syndrome and its impact on the elderly anticoagulated. Results and Discussion: Fragility has been termed to the percentage of elderly who were diagnosed with a greater deterioration of their biological, physical and cognitive systems, leading to a greater probability of adverse outcomes in health, disability and death. Some clinical features have been studied to identify patients as fragile; Linda Fried's frailty phenotype evaluates these characteristics; furthermore, there are strategies for monitoring oral anticoagulants that should be known to reduce the risk of adverse events. Conclusions: The frail elderly has some risk factors that are likely to affect the efficacy and safety of oral anti-clotting therapy. Existing clinical guidelines do not provide enough evidence and do not consider the elderly patient in a multidimensional manner. Cadena-Sanabria MO, Pinto-Saavedra OM, ContrerasValero JF, Ortega-Ramírez GE, Bautista-Mier HA. Oral anticlotting in the fragile elderly with atrial fibrillation. MedUNAB 2017; 19(3): 221-229]

Keywords: Atrial Fibrillation; Frail Elderly; Anticoagulants; Drug-Related Side Effects and Adverse Reactions; Geriatrics.

\section{Introducción}

La Fibrilación Auricular (FA) constituye la arritmia más frecuente en la práctica clínica, y su prevalencia está directamente relacionada con la edad (1). Esta representa la indicación más frecuente de anticoagulación crónica, seguida del tromboembolismo venoso (2). Los objetivos fundamentales de la anticoagulación oral son reducir la incidencia de eventos cardioembólicos y recurrencia de tromboembolismo, por tanto, disminuir la probabilidad de deterioro funcional, mortalidad y pobre calidad de vida (3).

Desafortunadamente, la evidencia científica deja de lado la población anciana más vulnerable al excluir, en la mayoría de estudios fase III, los pacientes muy mayores (por encima de 80 años de edad), con alta carga de comorbilidad (enfermedad renal y hepática avanzada), polifarmacia, deterioro cognitivo y dependencia funcional (Rankin modificado mayor de 3). Esto conlleva a que, en la práctica clínica diaria, los eventos adversos ante el uso de antico-

\section{Resumo}

Introdução: O envelhecimento aumenta o risco de trombose e embolias, traz mudanças fisiológicas e comorbidades como a fibrilhação auricular que fazem complexo o inicio e a segurança da anticoagulação. Apesar do aumento da disponibilidade das clínicas de anticoagulação e novos anticoagulantes orais, é baixa a percentagem de adultos mais velhos que tomam anticoagulantes ou que tenham a indicação completa e ausência de contra-indicações. Objetivo: Revisar as principais estratégias de prever e reduzir o risco de sangramento com o uso de anticoagulantes orais nos pacientes idosos mais frágeis. Metodologia: Se fez uma revisão sistemática de estudos que avaliaram a segurança e eficácia de anticoagulantes orais nos pacientes com fibrilhação auricular em adultos com mais de 65 anos, e uma busca dos estudos sobre o síndrome de fragilidade e o seu impacto nos idosos anticoagulado. Resultados e Discussão: Foi chamada de fragilidade a percentagem de pessoas idosas com maior deterioro de seus sistemas biológicos, físicos e cognitivos, levando-as a uma probabilidade maior de resultados adversos na saúde, invalidez e morte. Foram estudadas as características clínicas que identificam pacientes como pacientes frágil; o fenótipo de fragilidade de Linda Fried avalia estas características; além disto, existem estratégias adicionais de monitoramento de anticoagulantes orais que devem ser conhecidos para minimizar o risco de fatos adversos. Conclusões: Os idosos frágeis presentam fatores de risco que podem afectar a eficácia e segurança da terapia com anticoagulantes orais. As diretrizes clínicas existentes não são claras nem as provas são suficientes. Além disto, não consideram de forma multidimensional aos pacientes geriátricos. [Cadena-Sanabria MO, Pinto-Saavedra OM, Contreras-Valero JF, Ortega-Ramírez GE, Bautista-Mier HA. Anticoagulação oral nas pessoas idosas com fibrilhação auricular. MedUNAB 2017; 19(3): 221-229]

Palavras-chave: Fibrilação Atrial; Idoso Fragilizado; Anticoagulantes; Efeitos Colaterais e Reações Adversas Relacionados a Medicamentos; Geriatria.

agulantes cumarínicos y nuevos anticoagulantes orales (NACO) sean más frecuentes que los descritos en los ensayos clínicos.

Se plantea revisar de manera general los aspectos diferenciales en este grupo etario que pueden representar un mayor riesgo de sangrado, así como describir las principales recomendaciones antes de definir el inicio de una terapia anticoagulante en pacientes mayores de 65 años.

\section{Estrategia de búsqueda de literatura}

Se llevó a cabo una búsqueda sistemática de literatura sobre estudios que evaluaran la seguridad y eficacia de anticoagulantes orales en pacientes con fibrilación auricular, específicamente en adultos mayores de 65 años. Por otra parte, se realizó una búsqueda de estudios analíticos sobre el síndrome de fragilidad y su impacto en el adulto mayor anticoagulado. La búsqueda se llevó a cabo entre junio de 
2015 y junio de 2016; en las bases de datos PubMed, Lilacs, Google Académico y Scielo. No hubo restricción por año de realización de los estudios.

Los idiomas de búsqueda fueron inglés y castellano. Los estudios se identificaron en todos los motores de búsqueda con la siguiente estrategia: (frailty) OR (frail elderly) AND (atrial fibrillation) AND (oral anticoagulants) AND (bleeding riskscores).

Los autores excluyeron registros duplicados y examinaron títulos y abstracts de todos los estudios potencialmente relevantes para esta revisión. Se llevó a cabo el análisis de los textos completos y, aquellos estudios que cumplieron con los criterios de elegibilidad fueron incluidos en esta revisión.

\section{Envejecimiento y fragilidad}

El envejecimiento es un proceso heterogéneo, con una gran variedad en el grado de autonomía y función física que una persona puede tener respecto a otra de su misma edad. Dentro de esta variabilidad, aparece un grupo de personas que tienen un grado de función biológica menor, que se traduce en una mayor vulnerabilidad a distintos eventos adversos. En la medicina geriátrica se ha tratado de catalogar este grupo de personas como ancianos frágiles (4).

\section{¿Qué es la fragilidad clínica?}

El síndrome de fragilidad consiste en un estado clínico de mayor vulnerabilidad y susceptibilidad a la presentación de complicaciones y discapacidad ante estresores agudos, refleja una disminución de la reserva fisiológica y de los mecanismos de adaptación biológicos (5). Se ha tratado de definir fenotípicamente a través de los criterios de Linda Fried descritos en su estudio de salud cardiovascular(6). En Colombia, Gómez JF y Cols., realizaron una validación de estos criterios a saber: velocidad de la marcha lenta $(<0.8 \mathrm{~m} / \mathrm{seg})$, disminución de la fuerza de agarre $(<15 \mathrm{~kg} / \mathrm{f})$, nula actividad física, pérdida de peso $5 \%$ en el último año o IMC $<21$ y sensación de fatiga. Si se cumplen 3 o más de los 5 criterios se hace diagnóstico de estado de fragilidad (7).

Existen numerosas herramientas descritas para la detección de fragilidad, las cuales pueden ser empleadas en los algoritmos de toma de decisiones en el adulto mayor, una de ellas es la escala: Identification of Seniors at Risk (ISAR) (8). Fue desarrollada en Canadá en 1999 y ha sido validada en diferentes idiomas. Está compuesta por un formulario sencillo de realización rápida, que evalúa la presencia de factores riesgo. Un punto por cada pregunta descrita: ¿Requiere asistencia para realizar actividades cotidianas?, ¿Luego de ingreso hospitalario requiere más ayuda de la habitual?, ¿Ha presentado hospitalización por más de 2 noches en los últimos 6 meses?, ¿Tiene problemas visuales?, ¿Tiene problemas significativos con la memoria?, ¿Toma más de 3 medicamentos? Un resultado $>2$ es positivo e indicativo de mayor vulnerabilidad, la cual debe ser manejada multidisciplinariamente. Posee sensibilidad del 74\%, especificidad 54\% y área bajo la curva ROC de 0.70 para predicción de eventos adversos(9).

\section{Fragilidad y su papel en anticoagulación}

La fragilidad tiene un gran impacto en la salud por su fuerte asociación con comorbilidad, discapacidad, deterioro cognoscitivo y depresión. A pesar de ello, no existen estudios que evalúen la asociación de fragilidad en Colombia con el uso de anticoagulantes orales y menos que determinen la frecuencia de complicaciones como sangrado mayor incluido hemorragia intracerebral.

En una cohorte de 206 pacientes evaluados en una unidad de agudos de hospitales de tercer nivel en Sydney (Australia), compararon pacientes frágiles y no frágiles que usaban warfarina, en cuanto a indicación y consecuencias clínicas, con un seguimiento a 6 meses (10). En este estudio los pacientes frágiles fueron menos propensos a recibir warfarina al ingreso y al egreso del hospital $(p<0.001)$. Lo más importante es que esos pacientes tuvieron un mayor riesgo de infarto cerebral cardioembólico (12.3\% vs 3.9\%) RR 3.5 (95\% IC 1.0-12.0), y mayor mortalidad RR 2.8 (95\% IC 1.2-6.5). El riesgo de hemorragia mayor fue más alto, aunque sin significancia estadística (RR 1.5, 95\% IC 0.7-3.0).

El anciano frágil presenta factores de riesgo adicionales que probablemente afectan la eficacia y seguridad de la terapia con anticoagulantes (11). Las guías clínicas existentes no proveen la suficiente evidencia y no consideran de manera multidimensional el paciente geriátrico. Existen áreas identificadas que requieren una evaluación integral antes de iniciar o descontinuar una terapia anticoagulante: comorbilidades, polifarmacia, adherencia, demencia, funcionalidad, barreras para el automonitoreo, estado nutricional, riesgo de caídas y pobre expectativa de vida (12). Con la introducción de nuevos anticoagulantes en el mercado, se amplían las opciones de terapia; sin embargo, dependiendo del estado de fragilidad, la conducta puede ser difícil de tomar. La determinación de costo efectividad de una terapia anticoagulante en una persona mayor de 80 años es compleja. Ante dependencia funcional severa no hay datos que permitan suponer un beneficio explícito de prevenir un cardioembolismo en pacientes con nulo potencial de rehabilitación o reducida expectativa de vida.

La edad por sí sola no debe constituir un factor para rechazar o decidir anticoagulación en un adulto mayor con fibrilación auricular. Existen características que habitualmente no son tenidas en cuenta en escalas de riesgo de sangrado. Ellas dejan de lado dominios fundamentales en la valoración 
geriátrica. Como ejemplos claros están: la presencia de deterioro cognitivo avanzado, sin un soporte social favorable, lo cual puede impactar en adherencia y posibilidad de monitoreo cuidadoso con el uso de antagonistas de la vitamina K (AVK) (13). Los cuadros de inestabilidad y caídas recurrentes, pueden derivar en la mayor frecuencia de hematomas y riesgo de hemorragias intraparenquimatosas, si bien no son una contraindicación explícita al inicio de anticoagulación, requieren un abordaje multidisciplinario(14).

\section{Predicción de sangrado y monitoriza- ción con el uso de anticoagulantes}

El riesgo de sangrado en los pacientes ancianos frágiles está determinado por múltiples factores, incluyendo la edad, interacción medicamentosa, función renal, función hepática, estado nutricional, consumo de alimentos (vegetales) (15).

En la fibrilación auricular no valvular las guías de la sociedad europea de cardiología (2010) y canadiense (2012) recomiendan el uso de tres escalas para predecir el riesgo de sangrado: HAS-BLED, HEMORR2HAGES, y ATRIA (Tabla 1 y 2). La sociedad europea de trombosis y ritmo cardiaco (EHRA) recomienda aplicar la escala $H A S-B L E D$ por su mayor valor predictivo; además, documenta aquellos factores de riesgo que pueden ser modificados para disminuir el riesgo de sangrado (16-18).

Hay características asociadas a un mayor riesgo de sangrado tras el uso de antagonistas de la vitamina $\mathrm{K}$ (warfarina) dentro de las cuales están: edad avanzada $>60$ años, sexo femenino, diabetes mellitus, neoplasias, anemia, hipertensión (sistólica $>180$ o diastólica $>100$ $\mathrm{mmHg}$ ), alcoholismo agudo o crónico, enfermedad hepática, enfermedad renal; mala adherencia al tratamiento y/o asistencia médica, enfermedad cerebrovascular previa, presencia de lesiones sangrantes (5 o más microsangrados a nivel de RNM cerebral, leucoaraiosis), úlcera péptica, antecedente de hemorragia gastrointestinal, trastornos hematológicos (trombocitopenia), el uso concomitante de aspirina o clopidogrel, antiinflamatorios no esteroideos (AINES), antibióticos, amiodarona, estatinas, fibratos, inhibidores selectivos de la receptación de serotonina (ISRS), Inestabilidad del control del INR y el INR> 3.0;

Tabla 1. Escala HASBLED para predicción de riesgo de sangrado con Warfarina.

\begin{tabular}{clr}
\hline LETRA & FACTORES DE RIESGO & PUNTAJE \\
\hline H & Hipertensión no controlada TAS $>160 \mathrm{mmHg}$ & 1 \\
\hline A & Función hepática o renal anormal & 102 \\
\hline S & Ictus & 1 \\
\hline B & Sangrado & 1 \\
\hline L & INR lábil & 1 \\
\hline E & Edad $>65$ años & 102 \\
\hline D & Uso de fármacos/alcohol & 9 \\
\hline Máximo puntaje & &
\end{tabular}

Fuente: Adaptado de Roldán y Cols. ${ }^{16}$

Tabla 2. Interpretación Escala HASBLED para predicción de riesgo de sangrado.

\begin{tabular}{clr}
\hline PUNTAJE & RIESGO DE & RIESGO DE SANGRADO POR 100 \\
& SANGRADO & PACIENTES- AÑO \\
\hline $\mathbf{0}$ & Bajo riesgo & $1.13 \%$ \\
\hline $\mathbf{1}$ & Riesgo Intermedio & $1.02 \%$ \\
\hline $\mathbf{2}$ & Riesgo Intermedio & $1.88 \%$ \\
\hline $\mathbf{3}$ & Alto riesgo & $3.74 \%$ \\
\hline $\mathbf{4}$ & & $8.7 \%$ \\
\hline $\mathbf{5 - 9}$ & & Datos insuficientes \\
\hline
\end{tabular}

Fuente: Adaptado de Roldán y Cols. ${ }^{16}$ 
hemorragia grave precedente durante el tratamiento con warfarina con un INR en el intervalo terapéutico (19-21).

El control del INR es importante cuando se inicia manejo con antagonistas de la vitamina K (AVK). Previo al inicio de AVK es posible determinar la probabilidad de alcanzar con éxito el rango terapéutico (INR entre 2-3) mediante la escala SAMe-TT 2 R 2 (Tabla 3); un puntaje entre 0-1 sugiere una probabilidad alta de alcanzar el rango terapéutico, un puntaje $\geq 2$ indica baja probabilidad de llegar a un INR adecuado y pueden requerir alguna intervención adicional o cambio de terapéutica por nuevos anticoagulantes orales(22).

Se debe recalcar que existen diferentes cambios fisiológicos que se presentan con el envejecimiento y con más énfasis en el anciano frágil, se requiere una dosificación menor en $\mathrm{mg}$ para dosis total semanal de warfarina, comparado con el adulto joven. Esta reducción de la dosis es obligatoria en aquellos pacientes con hipoalbuminemia o peso menor de $50 \mathrm{~kg}(23)$.

\section{Los nuevos anticoagulantes orales}

Los nuevos anticoagulantes orales (NACO) son medicamentos con una acción farmacológica más estable, lo cual limita el riesgo de interacciones medicamentosas y evita la realización de una monitorización rutinaria estrecha de sus niveles plasmáticos, ventajas a destacar en comparación con la warfarina (24). Sin embargo, existen condiciones de urgencia en donde es relevante determinar el nivel de anticoagulación de un paciente. (Por ejemplo, cirugía de urgencia o el tratamiento de hemorragias mayores)(25).

- Para el Dabigatran se han establecido los siguientes parámetros: el tiempo de tromboplastina parcial activada (TTPa); Tiempo de trombina (TT) y el tiempo de coagulación con ecarina (TCE) (25). El TTPa permite determinar una evaluación cuantitativa del efecto anticoagulante. En promedio los pacientes 12 horas después de la última dosis de dabigatran presentan valores 1.5 veces el límite superior (50 segundos), menos de un $10 \%$ de los pacientes tienes valores mayores a 2 veces. Un TTPa mayor a 2 veces el control (70 segundos), sugiere anticoagulación excesiva(26). El tiempo de trombina Diluido (HEMOCLOT), permite definir cuantitativamente la concentración plasmática y la actividad anticoagulante, puede cuantificar concentraciones plasmáticas desde 100 - $200 \mathrm{nmol} / \mathrm{L}$; Una concentración de Dabigatran mayor $200 \mathrm{ng} / \mathrm{ml}$, se correlaciona con un Hemoclot mayor $65 \mathrm{~s}$; lo cual se asocia a un incremento del riesgo de sangrado. Se ha sugerido un rango de normalidad entre $(40-100 \mathrm{~s})$. El TCE, mide la actividad del dabigatran con una prolongación dosis lineal hasta $500 \mathrm{ng} / \mathrm{ml}$; un TCE más de 3 veces el límite superior normal, es asociado con alto riesgo de sangrado y se correlaciona con niveles plasmáticos de $(200-300 \mathrm{ng} / \mathrm{dl})(27,28)$.

- Para Apixaban y Rivaroxaban (inhibidores del factor Xa) el Tiempo de Protrombina (TP) y TTPa no son suficientes para determinar el nivel de anticoagulación. La prueba con mayor especificidad es la medición específica del antifactor Xa cromogénico; este permite cuantificar las concentraciones plasmáticas del medicamento y predecir el riesgo de sangrado (29).

\section{Elección de Anticoagulantes orales en adultos mayores}

Sardar y Cols., describieron en un metaanálisis de 25,031 pacientes mayores de 75 años, que el uso de NACO no causó exceso de sangrados e incluso fue igual o más

Tabla 3. Escala SAMe-TT 2 R 2 para predecir logro de INR terapéutico.

\begin{tabular}{clr}
\hline ACRÓNIMO & DEFINICIÓN & PUNTOS \\
\hline $\mathbf{S}$ & Sexo (femenino) & 1 \\
\hline $\mathbf{A}$ & Años (<60) & 1 \\
\hline $\mathbf{M}$ & Historia médica* & 1 \\
$\mathbf{e}$ & & 1 \\
\hline $\mathbf{T}$ & Tratamiento (Interacción medicamentosa, \\
& amiodarona) & 2 \\
\hline $\mathbf{T}$ & Tabaco (Durante dos años recientes) & 2 \\
\hline $\mathbf{R}$ & Raza negra & 8 \\
\hline Puntos máximos & & \\
\hline
\end{tabular}

* Más de dos de las siguientes: hipertensión, diabetes, enfermedad coronaria/Infarto del miocardio, enfermedad arterial periférica, falla cardiaca congestiva, antecedente de ACV, enfermedad pulmonar, y enfermedad hepática o renal. 
efectivo comparado con warfarina en pacientes con FA no valvular para prevención de cardioembolismo (dabigatran, apixaban) y para recurrencia de tromboembolismo venoso (TEV) así como muerte relacionada con TEV (30). Se presenta un resumen de la dosis recomendada para el grupo de pacientes frágiles respecto a la administración de NACO (Tabla 4).

Se presenta en una tabla la decisión que surge de elegir como primera opción NACO o warfarina, según lo recomendado por Edholm $\mathrm{K}$ y Cols., en su revisión sistemática (Tabla 5) (32). En dicha publicación se resalta la evidencia sólida a favor de los NACO respecto a la superioridad en la prevención del riesgo de sangrado intracerebral e inclusive mortalidad. Por tanto, se recomiendan como primera línea en pacientes con FA de origen no valvular. La elección de warfarina como primera opción sigue presente en el escenario de FA valvular (estenosis mitral severa o prótesis valvulares mecánicas) y en pacientes con falla renal avanzada (depuración de creatinina menor de $30 \mathrm{ml} / \mathrm{min}$ ).

\section{Conclusiones}

El clínico debe estar familiarizado con el proceso de envejecimiento y la condición de fragilidad, sobre todo en los pacientes adultos mayores que presentan FA y requieren un anticoagulante oral. Debe evitarse la discriminación por edad y se deben promover estrategias seguras de anticoagulación en este grupo de pacientes. La evaluación funcional y la determinación de síndromes geriátricos deben hacer parte habitual del enfoque de un adulto mayor con indicación de anticoagulación.

Es necesaria una cohorte que enrole números similares de pacientes frágiles, no frágiles con múltiples intervenciones (AVK, NACO y antiagregantes) para definir el papel de la fragilidad en la respuesta a la terapia y desenlaces clínicos. Existe incertidumbre al respecto en el ámbito internacional y regional, por tanto, se debe estimular el desarrollo de líneas de investigación en esta problemática. Por último, se presenta un algoritmo (Figura 1) a manera de propuesta que permite una evaluación más adecuada de anticoagulación en

Tabla 4. Posología adecuada de nuevos anticoagulantes (NACO) en ancianos frágiles con fibrilación auricular.

\begin{tabular}{cccc}
\hline Característica & $\begin{array}{c}\text { Dabigatran } \\
\text { (Pradaxa) }\end{array}$ & $\begin{array}{c}\text { Rivaroxaban } \\
\text { (Xarelto) }\end{array}$ & $\begin{array}{c}\text { Apixaban } \\
\text { (Eliquis) }\end{array}$ \\
\hline $\begin{array}{c}\text { Dosis } \\
\text { Recomendada }\end{array}$ & $110 \mathrm{mg} / 12$ horas & $15 \mathrm{mg} / \mathrm{día}$ & $2.5 \mathrm{mg} / 12$ horas \\
\hline Mecanismo & Inhibidor de la trombina & $\begin{array}{c}\text { Inhibidor directo del factor } \\
\text { Xa. }\end{array}$ & $\begin{array}{c}\text { Inhibidor directo del factor } \\
\text { Xa. }\end{array}$ \\
\hline Monitorización & TTPa, HEMOCLOT, TT & $\begin{array}{c}\text { TP, Antifactor Xa } \\
\text { cromogénico }\end{array}$ & $\begin{array}{c}\text { Antifactor Xa cromogénico } \\
\end{array}$ \\
\hline Excreción Renal & $85 \%$ & $33 \%$ Inactivo & $50 \%$ \\
\hline Dializable & $\mathrm{Si}$ & $\mathrm{No}$ & $\mathrm{Si}$ \\
\hline Transporte GP.P & $\mathrm{Si}$ & $\mathrm{Si}$ & $\mathrm{Si}$ \\
\hline Pico de Acción & $1.25-3$ horas & $2-4$ horas & $1-4$ horas \\
\hline
\end{tabular}

Fuente: Adaptado de Veiga y Cols. 31

Tabla 5. Preferencia de anticoagulante oral según características de paciente mayor.

\begin{tabular}{ll}
\hline Warfarina & Nuevos Anticoagulantes (NACO) \\
\hline Válvulas mecánicas & Historia de hemorragia intracerebral \\
\hline Deterioro renal severo eTFG $<30 \mathrm{ml} / \mathrm{min}$ & INR inestable, TTR<60\% \\
\hline Pobre adherencia a medicamentos & No deseo de cumplimiento de esquemas de dos \\
(mayor vida media de la warfarina) & dosis día (se prefiere Rivaroxaban) \\
\hline Limitación de costos & Interacciones farmacológicas frecuentes \\
\hline Historia de sangrado gastrointestinal (preferible & \\
Warfarina, Apixaban podría ser una opción) & Historia de Dispepsia (Se prefiere Apixaban) \\
\hline
\end{tabular}

Fuente: Adaptado de Edholm y Cols. 31 


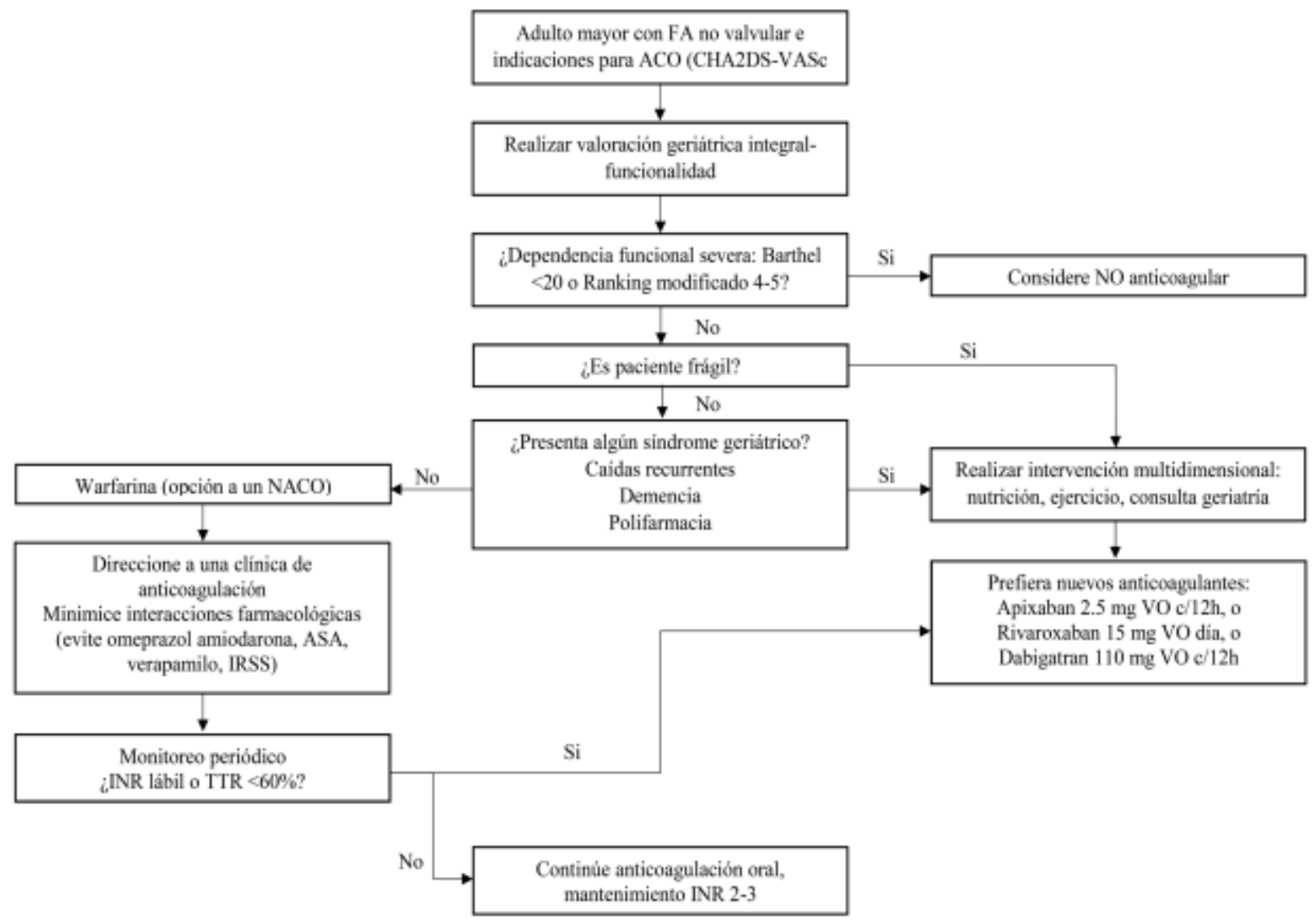

Fuente: Elaboración propia de los autores

Figura 1. Algoritmo sugerido para toma de decisiones en anticoagulación para mayores de 65 años. ACO: anticoagulación oral. NACO: nuevo anticoagulante oral. ASA: ácido acetilsalicílico. IRSS: inhibidor de recaptación de serotonina selectivo (ej: fluoxetina, sertralina). INR: índice normalizado internacional. TTR: tiempo de INR en rango terapéutico (INR entre 2 y 3 ) (33-37)

diferentes escenarios clínicos concernientes al adulto mayor.

\section{Conflicto de intereses}

Los autores manifiestan que no tienen ningún conflicto de interés.

\section{Referencias}

1. Sheikh A, Patel NJ, Nalluri N, Agnihotri K, Spagnola J, Patel A, Asti D, Kanotra R, Khan H, Savani C, Arora S, Patel N, Thakkar B, Patel N, Pau D, Badheka AO, Deshmukh A, Kowalski M, Viles-Gonzalez J, Paydak H. Trends in hospitalization for atrial fibrillation: epidemiology, cost, and implications for the future. Prog Cardiovasc Dis 2015; 58(2):105-116. Doi: 10.1016/j.pcad.2015.07.002

2. Wieloch $M$, Själander $A$, Frykman $V$, Rosenqvist $M$, Eriksson N, Svensson PJ. Anticoagulation control in
Sweden: reports of time in therapeutic range, major bleeding, and thrombo-embolic complications from the national quality registry AuriculA. Eur Heart J 2011; 32(18):2282-2289. Doi: 10.1093/eurheartj/ehr134

3. Olesen JB, Lip GY, Lindhardsen J, Lane DA, Ahlehoff O, Hansen ML, Raunsø J, Tolstrup JS, Hansen PR, Gislason $\mathrm{GH}$, Torp-Pedersen C. Risks of thromboembolism and bleeding with thromboprophylaxis in patients with atrial fibrillation: A net clinical benefit analysis using a 'real world' nationwide cohort study. Thromb Haemost 2011; 106(4):739-749. Doi: 10.1160/TH11-05-0364

4. Clegg A, Young J, lliffe S, Olde-Rikkert MGM, Rockwood K. Frailty in Older People. Lancet 2013; 381(9868):752762. Doi:10.1016/S0140-6736(12)62167-9

5. Rodríguez-Mañas L, Fried LP. Frailty in the clinical scenario. Lancet $2014 ; 385(9968)$ :e7-e9. Doi: 10.1016/S0140-6736(14)61595-6

6. Fried LP, Tangen CM, Walston J, Newman AB, Hirsch C Gottdiener J, Seeman T, Tracy R, Kop WJ, Burke G, McBurnie MA, Cardiovascular Health Study Collaborative Research Group. Frailty in Older Adults: Evidence for a Phenotype. J Gerontol A Biol Sci Med Sci 2001; 56A(3):M146-156. Doi: 10.1093/gerona/56.3.M146 
7. Gómez-Montes JF, Curcio-Borrero CL, Henao GM Fragilidad en ancianos Colombianos. Rev Medica.Sanitas 2012; 15(4): 8-16.

8. Cadena-Sanabria MO, Rodríguez-Arrieta LA, GallegoAndrade RG. Escala Identification of Seniors at Risk (ISAR): herramienta para la predicción de eventos adversos en el departamento de Urgencias. Rev Asoc Colomb Gerontol Geriatr 2013; 27(4):1887-1896.

9. McCusker J, Bellavance F, Cardin S, Trépanier S, Verdon $\mathrm{J}$, Ardman O. Detection of older people at increased risk of adverse health outcomes after an emergency visit: the ISAR screening tool. J Am Geriatr Soc 1999; 47(10):1229 1237. Doi: 10.1111/j.1532-5415.1999.tb05204.x

10. Perera V, Bajorek BV, Matthews S, Hilmer SN. The impact of frailty on the utilisation of antithrombotic therapy in older patients with atrial fibrillation. Age Ageing 2009; 38(2):156162. Doi: 10.1093/ageing/afn293

11. Fang MC, Chang Y, Hylek EM, Rosand J, Greenberg SM, Go AS, Singer DE. Advanced age, anticoagulation intensity, and risk for intracranial hemorrhage among patients taking warfarin for atrial fibrillation. Ann Intern Med 2004; 141(10):745-752. Doi: 10.7326/0003-4819-141-10200411160-00005

12. Fang MC, Go AS, Hylek EM, Chang Y, Henault LE, Jensvold NG, Singer DE. Age and the risk of warfarinassociated hemorrhage: the anticoagulation and risk factors in atrial fibrillation study. J Am Geriatr Soc 2006; 54(8):1231-1236. Doi: 10.1111/j.1532-5415.2006.00828.x

13. Froom $\mathrm{P}$, Miron $\mathrm{E}$, Barak M. Oral anticoagulants in the elderly. Br J Haematol 2003;120(3)526-528.

14. Gage BF, Birman-Deych E, Kerzner R, Radford MJ, Nilasena DS, Rich MW. Incidence of intracranial hemorrhage in patients with atrial fibrillation who are prone to fall. Am J Med 2005; 118(6):612-617. Doi: 10.1016/j.amjmed.2005.02.022

15. Rosand J, Eckman MH, Knudsen KA, Singer DE, Greenberg SM. The effect of warfarin and intensity of anticoagulation on outcome of intracerebral hemorrhage. Arch Intern Med 2004; 164(8):880-884. Doi: 10.1001/archinte.164.8.880

16. Roldán V, Marín F, Fernández H, Manzano-Fernández S, Gallego P, Valdés M, Vicente V, Lip GY. Predictive value of the HAS-BLED and ATRIA bleeding scores for the risk of serious bleeding in a "real-world" population with atrial fibrillation receiving anticoagulant therapy. Chest 2013; 143(1):179-184. Doi: 10.1378/chest.12-0608

17. Apostolakis S, Lane DA, Guo Y, Buller H, Lip GYH. Performance of the HEMORR2HAGES, ATRIA and HAS-BLED bleeding risk prediction scores in anticoagulated patients with atrial fibrillation: The AMADEUS study. J Am Coll Cardiol 2013; 61(3): 386 -389. Doi: 10.1016/j.jacc.2012.10.010

18. Lip GY, Andreotti F, Fauchier L, Huber K, Hylek E, Knight E, Lane D, Levi M, Marín F, Palareti G, Kirchhof P, European Heart Rhythm Association. Bleeding risk assessment and management in atrial fibrillation patients. Executive Summary of a Position Document from the European Heart Rhythm Association [EHRA], endorsed by the European Society of Cardiology [ESC] Working Group on Thrombosis. Thromb Haemost 2011; 106(6):997-1011. Doi: 10.1160/TH11-10-0690

19. Delaney JA, Opatrny L, Brophy JM, Suissa S. Drug drug interactions between antithrombotic medications and the risk of gastrointestinal bleeding. CMAJ 2007; 177(4):347351. Doi: $10.1503 / \mathrm{cmaj} .070186$

20. Fitzmaurice DA, Blann AD, Lip GY. Bleeding risks of antithrombotic therapy. BMJ 2002; 325(7368):828-831.

21. Schelleman H, Bilker WB, Brensinger CM, Wan F, Yang YX, Hennessy S. Fibrate/Statin initiation in warfarin users and gastrointestinal bleeding risk. Am J Med 2010;123(2):151-157. Doi: 10.1016/j.amjmed.2009.07.020

22. Apostolakis S, Sullivan RM, Olshansky B, Lip GY. Factors affecting quality of anticoagulation control among patients with atrial fibrillation on warfarin: the SAMe-TT ${ }_{2} \mathrm{R}_{\mathbf{2}}$ score.

Chest 2013; 144(5):1555-1563. Doi: 10.1378/chest.130054

23. Singla DL, Morrill GB. Warfarin maintenance dosages in the very elderly. Am J Health Syst Pharm 2005;62 (10):1062-1066.

24. Vogel T, Geny B, Kaltenbach G, Lang PO. Anticoagulation in atrial fibrillation in the elderly: the geriatrician point of view with a focus on the direct oral anticoagulants. Rev Med Interne 2015; 36(1):22-30. Doi: 10.1016/j.revmed. 2014.08.005

25. Wang $\mathrm{Y}$, Bajorek B. New oral anticoagulants in practice: pharmacological and practical considerations. Am J Cardiovasc Drugs 2014; 14(3):175-189. Doi: 10.1007/ s40256-013-0061-0

26. Heidbuchel H, Verhamme P, Alings M, Antz M, Hacke W, Oldgren J, Sinnaeve P, Camm AJ, Kirchhof P, European Heart Rhythm Association. European Heart Rhythm Association Practical Guide on the use of new oral anticoagulants in patients with non-valvular atrial fibrillation. Europace 2013; 15(5):625-651. Doi: 10.1093/ europace/ eut083

27. Gosselin RC, Dwyre DM, Dager WE. Measuring dabigatran concentrations using a chromogenic ecarin clotting time assay. Ann Pharmacother 2013; 47(12):16351640. Doi: $10.1177 / 1060028013509074$

28. Stangier J, Feuring M. Using the HEMOCLOT direct thrombin inhibitor assay to determine plasma concentrations of dabigatran. Blood Coagul Fibrinolysis 2012; 23(2):138-143. Doi: 10.1097/ MBC. 0b013e32834f1b0c

29. Gouin-Thibault I, Flaujac C, Delavenne X, Quenet S, Horellou MH, Laporte S, Siguret V, Lecompte T. Assessment of apixaban plasma levels by laboratory tests: suitability of three anti-Xa assays. A multicentre French GEHT study. Thromb Haemost 2014; 111(2):240-248. Doi: 10.1160/TH13-06-0470

30. Sardar P, Chatterjee S, Chaudhari S, Lip GY. New oral anticoagulants in elderly adults: evidence from a metaanalysis of randomized trials. J Am Geriatr Soc 2014; 62(5):857-864. Doi: 10.1111/jgs.12799

31. Veiga-Fernández F, Malfeito-Jiménez MDR, BarrosCerviño SM, Magariños-Losada MDM. La anticoagulación oral en el anciano con fibrilación auricular no valvular. Rev Esp Geriatr Gerontol 2015; 50(3):134-142. Doi:10.1016/ j.regg.2015.01.005

32. Edholm K, Ragle N, Rondina MT. Antithrombotic management of atrial fibrillation in the elderly. Med Clin North Am 2015; 99(2):417-430. Doi: 10.1016/j.mcna. 2014.11.012

33. Granziera S, Cohen AT, Nante G, Manzato E, Sergi G. Thromboembolic prevention in frail elderly patients with atrial fibrillation: a practical algorithm. J Am Med Dir Assoc 
2015; 16(5):358-64. Doi: 10.1016/j.jamda.2014.12.008

34. Lubitz SA, Bauer KA, Benjamin EJ, Besdine RW, Forman DE, Gurol ME, Reddy VY, Singer DE. Stroke prevention in atrial fibrillation in older adults: existing knowledge gaps and areas for innovation: a summary of an American Federation for Aging research seminar. J Am Geriatr Soc 2013; 61(10):1798-803. Doi: 10.1111/jgs.12456

35. Hylek EM, Evans-Molina C, Shea C, Henault LE, Regan $\mathrm{S}$. Major hemorrhage and tolerability of warfarin in the first year of therapy among elderly patients with atrial fibrillation.
Circulation 2007;115(21):2689-2696. Doi: 10.1161/ CIRCULATIONAHA.106.653048

36. Zarraga IG, Kron J. Oral anticoagulation in elderly adults with atrial fibrillation: integrating new options with old concepts. J Am Geriatr Soc 2013; 61(1):143-150. Doi: 10.1111/jgs.12042

37. Granger CB, Alexander JH, McMurray JJ, Lopes RD, Hylek EM, Hanna M, et al. Apixaban versus warfarin in patients with atrial fibrillation. N Engl J Med 2011; 365(11):981-992. Doi: 10.1056/NEJMoa1107039 